\title{
A study of Microalbuminuria and its association with various cardiovascular risk factors and complications in patients with Type II Diabetes Mellitus
}

\author{
Goswani $D^{1}$, Jatav $M^{2}, \operatorname{Raval} \mathbf{A}^{3}$ \\ ${ }^{1}$ Dr. Divyesh Goswami, Associate Professor, ${ }^{2}$ Dr. Manan Jadav, Assistant Professor, ${ }^{3}$ Dr. Abhishek Raval, Assistant \\ Professor; all authors are attached with Department of Pathology, Dr. M. K. Shah Medical College and Research Centre, \\ Chandkheda, Ahmedabad, Gujrat, India.
}

Address for Correspondence: Dr. Manan Jadav, Email: rockki1234@gmail.com

\begin{abstract}
Background: Microalbuminuria is a key risk factor for several cardiovascular diseases and renal impairment. This is particularly true for diabetics, in whom it is common and can be used to predict the development of complications. Materials and Methods: A prospective observational study. Participants were divided into 3 different groups: Group I Control $(n=20)$, Group II - Patients with type II diabetes mellitus ( $\mathrm{n}=300)$, Group III - Diabetic patients with retinal changes $(n=50)$. Medical history, physical examination, complete blood count, ECG, chest X-ray and urine examination, tests for microalbuminuria, echocardiogram and fluorescein angiography were performed. Results: Mean age of patients in group I and II were $57.60 \pm 11.07$ and $57.04 \pm 11.57$ years respectively. $46 \%$ participants were taking oral anti diabetic agents. Out of 300 patients of type 2 DM-64\% were micral test negative and 36\% were micral test positive. In group III, 32\% were normoalbuminuric and $68 \%$ were microalbuminuric. Positive correlation was found between duration of DM and presence of microalbuminuria. Serum cholesterol and serum triglycerides level were more in microalbuminurics than normoalbuminurics and the difference was statistically significant while the difference in the levels of serum LDL and serum HDL was statistically insignificant between two groups. 55.6\% of the diabetics with microalbuminuria were hypertensive. Conclusion: Microalbuminuria can be considered as one of the predictive factors for overt nephropathy and various complications of DM. Thus, detection of microalbuminuria at an early stage may help in preventing the diabetic complications.
\end{abstract}

Keywords: Microalbuminuria, Diabetes mellitus, Dyslipidemia, hypertension

\section{Introduction}

Diabetes mellitus (DM), the most common endocrine disease, is a metabolic syndrome, characterized by chronic hyperglycemia and disturbances of carbohydrate, fat and protein metabolism associated with absolute or relative deficiency in insulin secretion and/or insulin action. The two main types of diabetes are type I or Insulin Dependent Diabetes Mellitus (IDDM) and type II or Non-Insulin Dependent Diabetes Mellitus (NIDDM). The various complications of diabetes mellitus are: Peripheral neuropathy; Microvascular disease and related abnormalities, which include diabetic nephropathy and diabetic retinopathy;

Manuscript received: $14^{\text {th }}$ April 2017

Reviewed: $25^{\text {th }}$ April 2017

Author Corrected: $4^{\text {th }}$ May 2017

Accepted for Publication: $11^{\text {th }}$ May 2017
Macrovascular disease with the influence of lipids, which include atherosclerosis, ischemic heart disease and cerebral stroke. The major cause of vascular dysfunction is hyperglycemia. Diabetic patients with poor glycemic control and longer duration of disease are more prone to develop various complications.

A correlation may exist between various complications of diabetes mellitus like microalbuminuria and overt nephropathy, retinopathy, ischemic heart disease, cerebral stroke etc and duration of diabetes and genetic predisposition. The presence of microalbuminuria, which can be detected by a simple, quick and noninvasive test, may be a predictor of vascular complications in patients with diabetes mellitus. Among 
Research Article

those with microalbuminuria, ischemic heart disease was more common. Metcalf et al concluded in their prospective studies in patients with type 2 diabetes mellitus that microalbuminuria was found in $37 \%$. They also concluded that albuminuria had significant relationship with diastolic blood pressure, body mass index, hypertriglyceridemia and hypercholesterolemia [1].

With this background, this study was carried out to find out the incidence of microalbuminuria in patients with type 2 diabetes mellitus and relation of microalbuminuria with duration of diabetes, lipid profile and presence of other complications like IHD / retinopathy /cerebral stroke/ neuropathy etc. An endeavor was made to evaluate whether presence of microalbuminuria in a diabetic patient has any predictive value for diagnosis of various complications.

\section{Materials and Methods}

Study design: Prospective and observational

Study setting: B J Medical College, Ahmedabad

Study duration: two years.

The study was commenced after approval from institutional ethical committee.

\section{Exclusion Criteria}

The following patients were excluded from the study:

1. Patients with macroalbuminuria i.e. urinary albumin excretion rate $>300 \mathrm{mg} /$ day or presence of other renal disease. Recent heavy exercise, use of NSAIDS, bacterial meningitis and UTI may give a false test for presence of albuminuria.

2. Patients with retinopathy due to other causes like hypertension, preeclampsia, eclampsia of pregnancy, blood dyscrasias and AIDS.

3. Patients having evidence of overt cardiovascular diseases including coronary artery disease and/or congestive heart failure ruled out by past and family history, smoking, hypertension, hypoestrogenemia in women, primary dyslipidemias.

The subjects included in the study were grouped as follows:

\section{Group I-Control group $(\mathbf{n}=\mathbf{2 0})$}

This group consisted of age, sex and BMI matched healthy subjects without any family history of diabetes.
They were selected from medical or paramedical staff, attendants of patients and general public.

\section{Group II-Diabetes group $(\mathbf{n}=\mathbf{3 0 0})$}

This group consisted of patients of type 2 diabetes mellitus.

\section{Group-III-Diabetes group with retinal changes $(\mathbf{n}=\mathbf{5 0})$}

All the subjects included in the study were subjected to detailed history, including family history of diabetes or IHD and a thorough physical examination along with relevant anthropometric data like BMI.Special emphasis was given on glycemic control, duration of diabetes, presence of hypertension, serum lipid levels and complications of diabetes. The criteria for presence of hypertension in various subjects were based on JNC VII (Joint National Committee) classification.

The following investigations were done:

1. Hemoglobin in gram \% (Cyanmethemoglobin method).

2. Total leukocyte count.

3. Differential leukocyte count.

4. Platelet count

5. ESR

6. Complete urine examination.

7. Blood glucose (Fasting and post prandial, by glucose oxidase-peroxidase method).

8. Blood urea.

9. Serum creatinine.

10. Lipid profile (serum Cholesterol, TG, VLDL, LDL and HDL).

11. Fundus examination.

12. Chest X-ray.

13. 12-lead ECG

\section{Special Investigations:}

1. Urinary albumin excretion rate measured in morning urine sample using sulphosalicylic acid test method and micral test by immunoassay strips (Boehringer Mannheim, Germany).

It is a dipstick test for detection of Microalbuminuria in 24 hour urine sample. The test is based on immunological detection of human albumin by means of soluble antibody- gold conjugate. Excess conjugate is retained in a separate zone containing immobilized human albumin. 


\section{Procedure}

1. The test strip is immersed in the urine and withdrawn after 5 seconds and is placed across the top of the urine vessel. When immersing and withdrawing the test strip, the test strip is not allowed to touch the collector vessel.

2. After 1 Minute, the color of test pad above the inscription "Micral" is compared with the color scale on the test strip container label. Comparison of the reaction color with the color scale is possible for another 5 minutes as the color is stable for that period.

Evaluation-Reaction colors lighter than the color block corresponding to approximately $20 \mathrm{mg} / \mathrm{L}$ albumin indicates a physiological urine albumin concentration. For determination of albumin concentration above $100 \mathrm{mg} / \mathrm{lit}$, the urine sample can be diluted by mixing one part of urine with two parts of water. The original albumin concentration is then calculated by multiplying the result obtained by 3 .

Color Development- The results are compared with the color scale on the test strip container label.

\begin{tabular}{|c|c|}
\hline No Change in color & Negative \\
\hline Light pink & $<20 \mathrm{mg} / \mathrm{L}$ \\
\hline Pink & $20-50 \mathrm{mg} / \mathrm{L}$ \\
\hline Dark pink & $50-100 \mathrm{mg} / \mathrm{L}$ \\
\hline
\end{tabular}

1. Urinary albumin excretion rate in morning urine sample using turbidimetric immunoassay.

2. Fluorescein angiography by dye sodium fluorescinate in patients having ophthalmoscopic changes of retinopathy on fundus examination.

3. Echocardiogram in patients having evidence of ischemic heart disease.

All the above measurements were taken in statistical analysis.

\section{Results}

In the present study, 20 healthy subjects (control group, group I) and 300 patients with diabetes mellitus type 2 (patient group, group II) were taken. In group I, there were 13 males and 7 females, while in Group II, the male and female patients were equal in number (150 patients each). The maximum patients (34\%) were in 61-70 years age group. Only $6 \%$ patients were more than 70 years of age. 50 patients of type $2 \mathrm{DM}$ were having retinal complications (Group III). In group III, there were 28 males and 22 females. All subjects were more than 40 years of age.

The age range of subjects in Group I (healthy controls) was 41-76 years and the mean age was $57.60 \pm 11.07$ years while it was 41-74 years with mean age $57.04 \pm 11.57$ years in Group II (diabetic patients). The mean age in males was slightly less than females in either groups but the difference was not statistically significant $(\mathrm{P}>0.05)$. The mean BMI was $22.9 \pm 2.7$ in group I and $24.6 \pm 2.45$ in group II and the difference was statistically insignificant $(\mathrm{P}>0.05)$. The mean body mass index was slighter higher in females than males $23.4 \pm 2.8 \mathrm{v} / \mathrm{s} 24.4 \pm 2.5$ in group II.

The most common symptoms at presentation in the diabetic patients were non-specific like headache, weakness, loss of appetite etc $(46 \%)$ followed by numbness/burning sensation in limbs (36\%), vomiting/abdominal pain/fever (20\%), vision problems $(12 \%)$, chest pain $(10 \%)$ and neurological deficit $(8 \%)$. Out of 300 patients, 48(16\%) were on diet therapy alone. Maximum patients $138(46 \%)$ were taking oral anti diabetic agents. 66(22\%) patients were on insulin alone while $48(16 \%)$ patients were receiving combination therapy (i.e. oral agents + insulin). Among them, most of the younger patients $(<60$ years) were on diet alone or taking oral drugs while elderly patients $(>60$ years) were mainly on insulin or combination therapy (insulin + oral agents).

The mean fasting blood glucose levels were $82.94 \pm 13.64 \mathrm{mg} / \mathrm{dl}$ and $186.55 \pm 73.43 \mathrm{mg} / \mathrm{dl}$ in group I and group II respectively. The difference was statistically highly significant $(\mathrm{P}<0.001)$. The mean post - prandial blood glucose levels were $118 \pm 15.44 \mathrm{mg} / \mathrm{dl}$ and $253.45 \pm 82.40 \mathrm{mg} / \mathrm{dl}$ in group I and group II respectively. The difference was statistically highly significant $(\mathrm{P}<0.001)$. 
Table-1 shows that all the healthy subjects were micral test negative i.e. were normoalbuminuric. Out of 300 patients of type 2 diabetes mellitus - $192(64 \%)$ were normoalbuminuric (micral test negative) and $108(36 \%)$ patients were microalbuminuric (micral test positive). There were equal numbers of male and female patients in both the subgroups of group II. Out of 50 patients of type 2 diabetes mellitus with retinal complications - $16(32 \%)$ were normoalbuminuric and $34(68 \%)$ were microalbuminuric. Conversely, prevalence of diabetic retinopathy was significantly more in microalbuminuric $(66.6 \%)$ than in normoalbuminurics $(18.7 \%)$ ( $\mathrm{P}$ value $<0.05)$.

Table-1: Presence of microalbuminuria in subjects studied.

\begin{tabular}{|c|c|c|c|c|c|c|c|c|c|}
\hline & \multicolumn{3}{|c|}{ Group I } & \multicolumn{3}{c|}{ Group II } & \multicolumn{3}{c|}{ Group III } \\
\hline & Male & Female & Total & Male & Female & Total & Male & Female & Total \\
\hline $\begin{array}{c}\text { Micral test } \\
\text { Negative }\end{array}$ & 13 & 7 & 20 & 96 & 96 & 192 & 10 & 6 & 16 \\
\hline $\begin{array}{c}\text { Micral test } \\
\text { Positive }\end{array}$ & 0 & 0 & 0 & 54 & 54 & 108 & 18 & 16 & 34 \\
\hline
\end{tabular}

The mean age was more in microalbuminurics $(56.7 \pm 9.0$ years $)$ than normoalbuminurics $(45.1 \pm 8.0$ years $)$ and the difference was statistically significant $(\mathrm{P}<0.05)$ while the mean BMI was also more in microalbuminurics $(25.6$ vs 24.2$)$ but the difference was not statistically significant $(\mathrm{P}>0.05)$. The mean duration of diabetic mellitus was significantly higher in microalbuminuric $(5.1 \pm 2.0$ years) group than in normoalbuminuric $(2.8 \pm 1.0$ years $)$ group $(\mathrm{P}<0.05)$. Table -2 shows that there is positive correlation between duration of diabetes mellitus and presence of microalbuminuria.

Table-2: Correlation of duration of diabetes and microalbuminuria.

\begin{tabular}{|c|c|c|}
\hline $\begin{array}{c}\text { Duration Of Diabetes } \\
\text { Mellitus(Years) }\end{array}$ & $\begin{array}{c}\text { Group II B (Micral Test } \\
\text { Positive) }\end{array}$ & $\begin{array}{c}\text { Coefficient Of } \\
\text { Correlation(r) }\end{array}$ \\
\hline$<5$ Years & 42 & 0.4 \\
\hline $5-10$ Years & 66 & 0.7 \\
\hline$>10$ Years & - & - \\
\hline
\end{tabular}

Table-3 shows that the difference in the lipid levels of serum cholesterol $(165.0 \pm 25.0 \mathrm{v} / \mathrm{s} 212.6 \pm 27.5)$ and serum triglycerides $(110.0 \pm 51.0 \mathrm{v} / \mathrm{s} 152.3 \pm 52.5)$ was statistically significant $(\mathrm{P}<0.05)$ between controls and diabetic patients. However, the serum LDL $(95.0 \pm 15.0 \mathrm{v} / \mathrm{s} 130.0 \pm 31.3)$ and serum HDL $(48.0 \pm 3.6 \mathrm{v} / \mathrm{s} 45.5 \pm 6.9)$ levels are statistically insignificant between two groups $(\mathrm{P}>0.05)$.

Table-4 shows that the serum cholesterol $(198.0 \pm 22.0 \mathrm{v} / \mathrm{s} 227.2 \pm 33.0)$ and serum triglycerides level $(135.7 \pm 49.0 \mathrm{v} / \mathrm{s}$ $169.0 \pm 56.0)$ were more in microalbuminurics than normoalbuminurics and the difference was statistically significant $(\mathrm{P}$ $<0.05)$ while the difference in the levels of serum LDL $(125.0 \pm 24.3 \mathrm{v} / \mathrm{s} 135.0 \pm 38.4)$ and serum HDL $(45.7 \pm 7.4 \mathrm{v} / \mathrm{s}$ $45.4 \pm 6.4)$ was statistically insignificant between two groups $(\mathrm{P}>0.05)$.

Table-3: Comparison of serum lipid in subjects studied.

\begin{tabular}{|c|c|c|c|}
\hline Serum Lipid & Group I \\
$\begin{array}{c}\text { Level } \\
(\mathbf{m g} / \mathbf{d l})\end{array}$ & $\begin{array}{c}\text { Group II } \\
\text { (Control) }\end{array}$ & $\begin{array}{c}\text { P-Value } \\
\text { (Diabetics) }\end{array}$ & \\
\hline (Mean + SD) & & & $\mathrm{P}<0.05$ \\
\hline Serum Cholesterol & $165+25$ & $212.6+27.5$ & $\mathrm{P}<0.05$ \\
\hline Serum Triglyceride & $110+51$ & $152.3+52.5$ & $\mathrm{P}>0.05$ \\
\hline Serum LDL & $95+15$ & $130.0+31.3$ & $\mathrm{P}>0.05$ \\
\hline Serum HDL & $48+3.6$ & $45.5+6.9$ & \\
\hline
\end{tabular}


Research Article

Table-4: Comparison of serum lipid levels in diabetic patients studied.

\begin{tabular}{|c|c|c|c|}
\hline $\begin{array}{c}\text { Serum Lipid } \\
\text { Level(mg/dl) } \\
\text { Mean }+ \text { SD) Serum }\end{array}$ & $\begin{array}{c}\text { Group IIA } \\
\text { Micral Test } \\
\text { Negative }\end{array}$ & $\begin{array}{c}\text { Group IIB } \\
\text { Micral Test } \\
\text { Positive }\end{array}$ & $\begin{array}{c}\text { P-Value } \\
\text { (IIA V/S IIB) }\end{array}$ \\
\hline Cholesterol & $198.0+22.0$ & $227.2+33.0$ & $<0.05$ \\
\hline Serum Triglyceride & $135.7+49.0$ & $169+56.0$ & $<0.05$ \\
\hline Serum LDL & $125.0+24.3$ & $135.0+38.4$ & $>0.05$ \\
\hline Serum HDL & $45.7+7.4$ & $45.4+6.4$ & $>0.05$ \\
\hline
\end{tabular}

Table-5 shows that in diabetic patients with negative micral test, most of the patients (75\%) had normal serum cholesterol levels of less than $200 \mathrm{mg} / \mathrm{dl}$ while in patients with positive micral test, $(50 \%)$ had high serum cholesterol levels range of $(200-240 \mathrm{mg} / \mathrm{dl})$. Most of the diabetic patients with either normoalbuminuria or microalbuminuria, had serum triglycerides levels $<150 \mathrm{mg} / \mathrm{dl}$, serum LDL levels less than $160 \mathrm{mg} / \mathrm{dl}$ and serum HDL levels more than $45 \mathrm{mg} / \mathrm{dl}$ which was not significant statistically $(\mathrm{P}>0.05)$ while in remaining patients serum triglycerides were more than $150 \mathrm{mg} / \mathrm{dl}$, serum LDL levels were more than $160 \mathrm{mg} / \mathrm{dl}$ and serum HDL levels were less than $35 \mathrm{mg} / \mathrm{dl}$ which was statistically significant $(\mathrm{P}<0.05)$.

Table-5: Relationship of lipid levels with Microalbuminuria in diabetic patients studied.

\begin{tabular}{|c|c|c|c|}
\hline & $\begin{array}{c}\text { Group II A } \\
\text { Micral test Negative }\end{array}$ & $\begin{array}{c}\text { Group II B } \\
\text { Micral test Positive }\end{array}$ & P-Value \\
\hline \multicolumn{4}{|c|}{ Serum cholesterol levels (mg/dl) } \\
\hline$<200$ & $144(75 \%)$ & $36(33.3 \%)$ & $\mathrm{P}>0.05$ \\
\hline $200-240$ & $42(21.9 \%)$ & $54(50 \%)$ & $\mathrm{P}<0.05$ \\
\hline$>240$ & $6(3.1 \%)$ & $18(16.7 \%)$ & $\mathrm{P}<0.05$ \\
\hline \multicolumn{4}{|c|}{ Serum triglyceride levels $(\mathrm{mg} / \mathrm{dl})$} \\
\hline$<150$ & $144(75 \%)$ & $48(44.4 \%)$ & $\mathrm{P}>0.05$ \\
\hline $150-200$ & $30(15.6 \%)$ & $36(33.3 \%)$ & $\mathrm{P}<0.05$ \\
\hline$>200$ & $18(9.4 \%)$ & $24(22.3 \%)$ & $\mathrm{P}<0.05$ \\
\hline \multicolumn{4}{|c|}{ Serum LDL levels $(\mathrm{mg} / \mathrm{dl})$} \\
\hline$<130$ & $132(68.7 \%)$ & $48(44.4 \%)$ & $\mathrm{P}>0.05$ \\
\hline $130-160$ & $48(25 \%)$ & $42(38.9 \%)$ & $\mathrm{P}<0.05$ \\
\hline$>160$ & $12(6.3 \%)$ & $18(16.7 \%)$ & $\mathrm{P}<0.05$ \\
\hline \multicolumn{4}{|c|}{ Serum HDL levels $(\mathrm{mg} / \mathrm{dl})$} \\
\hline$>45$ & $66(34.4 \%)$ & $36(33.3 \%)$ & $\mathrm{P}>0.05$ \\
\hline $45-35$ & $108(56.2 \%)$ & $60(55.6 \%)$ & $\mathrm{P}<0.05$ \\
\hline$<35$ & $18(9.4 \%)$ & $12(11.1 \%)$ & $\mathrm{P}<0.05$ \\
\hline
\end{tabular}

Table-6: Correlation of blood pressure with microalbuminuria in diabetic patients studied.

\begin{tabular}{|c|c|c|c|}
\hline $\begin{array}{c}\text { Blood } \\
\text { Pressure(mmHg) }\end{array}$ & $\begin{array}{c}\text { Group IIA } \\
\text { Micral Test } \\
\text { Negative (\%) }\end{array}$ & $\begin{array}{c}\text { Group IIB } \\
\text { Micral Test } \\
\text { Positive (\%) }\end{array}$ & P-Value \\
\hline $\begin{array}{c}\text { Normotensive } \\
\text { Mild to Moderate }\end{array}$ & $132(68.7 \%)$ & $48(44.4 \%)$ & $\mathrm{P}>0.05$ \\
\hline Hypertensive Severe & $42(21.9 \%)$ & $30(27.8 \%)$ & $\mathrm{P}<0.05$ \\
\hline Hypertensive & $18(9.3 \%)$ & $30(27.8 \%)$ & $\mathrm{P}<0.05$ \\
\hline
\end{tabular}

Table-6 shows that in diabetic patients with normoalbuminuria, most of the patients $(68.8 \%)$ were normotensives while in microalbuminurics, most of the patients $(55.6 \%)$ were hypertensive. 


\section{Discussion}

The present study was conducted on 20 - age, sex, and body mass index (BMI) matched healthy controls (Group I) and 300 patients of type 2 diabetes mellitus (Group II). The mean age was slightly more in females as compared to males. The apparent increase in mean age of females may be incidental finding or may be due to increased life expectancy in females. The mean BMI was similar in controls and diabetic patients.

Out of 300 patients studied, Most of the younger patients ( $\leq 60$ years) were on diet therapy or taking oral agents alone while elderly patients ( $>60$ years) were mainly on insulin or combination therapy. Insulin requirement increased as duration of diabetes increased. In the present study also, the number of patients on insulin increased with the advancing age which probably reflects duration of illness, gradual reduction in beta cell mass, failure of oral anti diabetic agent and need for better glycemic control.

The presence of microalbuminuria was measured in all the subjects studied. Among the diabetic patients, 108 (36\%) patients were micral positive. The diabetic population at risk may now be identified fairly accurately by the detection of microalbuminuria which strongly predicts the development of diabetic nephropathy [2]. Many workers in these studies showed that 21 of 26 diabetic patients in whom diabetic nephropathy would have developed could be identified by one measurement of microalbuminuria (predictive power of $80 \%$ ). Recently, it has been shown, those diabetics with elevations in urinary albumin excretion have a rather slow but notably yearly increase in albumin excretion rates: about $25 \mu \mathrm{g} / \mathrm{min} / \mathrm{year}$. Thus it can be concluded that increasing urinary albumin excretion is a strong predictor of the development of overt nephropathy.

The chronic effect of glucose in inducing tissue injury may occur via advanced glycated end products (AGE), polyol pathway, and protein kinase $C \beta-11$ [3]. All these products may lead to decreased charge of GBM, loss of glomerular fixed negative charges, extracellular matrix linking and activation of cytokines.

The prevalence of microalbuminuria in the present study is $36 \%$ which is comparable to the findings reported by Fiad et al [4]. However Gupta et al reported a lower prevalence of $26.6 \%$ in 65 type 2 North Indian diabetic patients [5]. These variations in prevalence can be attributed to factors such as racial difference, definition of microalbuminuria, method of urine collection etc. However, this could also reflect a true difference in the ethnic susceptibility to nephropathy.

A comparison of age and anthropometric measurements was done amongst micral positive and micral negative patients. In the present study, the mean age of microalbuminuric patients was more than the normoalbuminuric patients and it was statistically significant. It is comparable to the studies by Collin et al who observed progressive increase in the prevalence of microalbuminuria with advancing age [6]. The mean duration of diabetes mellitus in patients with microalbuminuria was more than in patients with normoalbuminuria. The coefficient of correlation also showed positive association between duration of diabetes mellitus and presence of microalbuminuria. The duration of diabetes influences the development of microvascular complications and patients with long standing diabetes have microalbuminuria.

Lipoprotein abnormalities are strongly associated with diabetic nephropathy. In the present study, the levels of serum cholesterol, serum triglycerides and serum LDL were higher and serum HDL were lower in control group than diabetic group. Also, the levels of serum cholesterol, serum triglycerides and serum LDL were higher and serum HDL were lower in microalbuminurics than normoalbuminurics. Park et al also reported significantly higher serum cholesterol and serum triglycerides levels in microalbuminurics as compared to normoalbuminuric diabetic patients [7].

There was no significant difference in serum LDL and HDL levels between healthy controls $\mathrm{v} / \mathrm{s}$ diabetic patients or normoalbuminuric v/s microalbuminurics. This shows that there is no consistent change in LDL or HDL concentrations in type 2 diabetic patients with or without microalbuminuria and they may be similar to those in healthy control individuals.

In the present study a positive association has been found between hypercholesterolemia and microalbuminuria. The incidence of microalbuminuria showed an increased trend with increasing serum cholesterol. A positive correlation between UAE rate and serum cholesterol has been demonstrated by Klein 
Research Article

et al who found elevated levels of total cholesterol in microalbuminuric diabetic patients. Hillege et al found that the concentration of cholesterol during a five year follow up period was positively related with the subsequent increase in urinary albumin excretion in microalbuminuric patients with type 2 diabetes [8].

Cholesterol is the main lipid component of LDL and HDL. An elevated level of cholesterol is the usual cause of increase in LDL concentration in the presence of relatively normal plasma triglycerides. Early diabetic nephropathy can itself influence lipoprotein metabolism by affecting urinary lipid excretion or by secondary effect on hepatic production and clearance of lipoprotein. Elevated serum cholesterol levels can accelerate endothelial cell damage and cause cholesterol ester accumulation and muscle cell proliferation, thus setting up a vicious cycle of ongoing microvascular damage.

In the present study an increased prevalence of microalbuminuria was found with increasing serum triglyceride levels. Sarnak et al stated that hypertriglyceridaemia is the hyperlipidiaemia par excellence of the diabetes [9]. Sukhija et al supported this statement with prevalence rates for hypertriglyceridaemia ranging from $20-50 \%$ [10].

Thus, in diabetic patients an elevation of triglyceride rich lipoproteins, chylomicrons and very low density lipoproteins (VLDL) is associated with increased levels of remnant lipoproteins and triglyceride enrichment of LDL and HDL. Increased triglycerides are more common in diabetic individuals with atherosclerotic disease and are more strongly associated with frequency of coronary artery disease (CAD) in diabetic individuals than is total serum cholesterol. This risk doubled in patients with diabetic nephropathy.

A striking positive association of urinary albumin excretion (UAE) with serum cholesterol and triglyceride levels but not with LDL has emerged from the present study which indicates a role of certain lipoprotein fractions in the pathogenesis and progression of diabetic microvascular disease but whether any fraction has a protective role remains unknown.

In the present study, no significant difference was found between prevalence of microalbuminuria or urinary albumin excretion rate with respect to metabolic control on HDL. Yuyun et al found that serum HDL cholesterol was similar at baseline and after follow-up in patients with persistently normal UAE and in those in whom microalbuminuria developed, whereas patients with raised baseline UAE had slightly lower concentration [11]. This strongly suggests that microalbuminuria develops before serum HDL cholesterol decreases; urinary loss of apolipoproteins has been implicated as a possible explanation.

The importance of the association between elevated blood pressure and UAE rate has been well recognized. As the blood pressure increases, the prevalence of microalbuminuria increases as found in the present study. Mykkanen et al also reported the prevalence of microalbuminuria as $60.5 \%$ in hypertensives and $3.6 \%$ in normotensives [12]. The association of pathologic UAE rate with rising blood pressure in diabetic patients appears to be secondary to the renal involvement. Microalbuminuria is also associated with abnormal circadian pattern of blood pressure. Both systolic and diastolic blood pressure is found to be significantly higher in patients with microalbuminuria.

Savage et al concluded that increasing UAE is associated with peripheral neuropathy, retinopathy, and cardiovascular disease in patients with diabetes [2]. A study conducted by Manaviat et al revealed that diabetic retinopathy was significantly related to albumin excretion [13]. Skyler et al suggested that at some stage during development of nephropathy, retinopathy almost inevitably occurs [14]. Since microalbuminuria predicts nephropathy, it should also predict retinopathy.

Albuminuria is a consequence of altered glomerular capillary membrane permeability, most often in the setting of diabetes and hypertension. The mechanisms for the association between microalbuminuria and increased cardiovascular disease risk are poorly understood, but several explanations have been proposed. Microalbuminuria may be a marker of widespread vascular damage [15]. Alternatively microalbuminuria may be a surrogate for other abnormalities that promote the development of cardiovascular complications, including insulin resistance and abnormalities of thrombogenic factors.

It is evident from the present study that microvascular and macrovascular complications of diabetes mellitus should be considered as a part of natural history of the disease and should be suspected and treated 
aggressively at an earlier stage to reduce morbidity and also mortality to a great extent. In the present study, presence of microalbuminuria was found to be one of the predictive factors for various complications but this is insufficient to explain the whole spectrum of disease. The pathogenesis is multifactorial and various causative factors like oxidative stress, abnormal cytokine production, increase lipid peroxidation, racial/ethnic susceptibility, glycemic status and other accompanying circumstances like obesity, preexisting renal disease have been held responsible for various vascular complications of diabetes mellitus.

It is difficult to assess incidence and prevalence of various complications in the patients with type 2 diabetes mellitus in a small study but simultaneous inclusion of age, sex and BMI matched controls and analyzing the statistical difference between two groups at $5 \%$ probability levels, there are fewer chances for these results being a chance association.

However, further elucidation is required in large cohort studies to draw conclusion on causation of factors responsible for various complications in patients with type 2 diabetes mellitus and their individual predictive value.

In conclusion, microalbuminuria can be considered as forerunner of overt diabetic nephropathy, coronary artery disease and cerebrovascular disease. It is similarly associated with other microvascular complications including diabetic retinopathy and macular edema, glomerular injury, endothelial dysfunction, and also insulin resistance. Poor glycemic control, dyslipidemia and high blood pressure are identified as risk factors for the development of microalbuminuia.

Thus, it can be concluded that detection of microalbuminuria at an early stage in diabetic patients is very helpful in preventing these complications.

Further studies proving the role of microalbuminuria as an indicator of diabetic complications should be conducted with the use of advanced methods to detect albuminuria accurately. This can serve as a cornerstone in the routine use of microalbuminuria as a predictor of the diabetic complications and hence can guide the precise management of the diabetic patients.

Funding: Nil, Conflict of interest: None initiated, Permission from IRB: Yes

\section{References}

1. Metcalf P, Baker J, Scott A, Wild C, Scragg R, Dryson E. Albuminuria in people at least 40 years old: effect of obesity, hypertension, and hyperlipidemia. Clinical chemistry. 1992; 38(9):1802-8.

2. Savage S, Estacio RO, Jeffers B, Schrier RW. Urinary albumin excretion as a predictor of diabetic retinopathy, neuropathy, and cardiovascular disease in NIDDM. Diabetes Care. 1996 Nov;19(11):1243-8.

3. Stehouwer CD, Nauta JJ, Zeldenrust GC, Hackeng WH, Donker AJ, den Ottolander GJ. Urinary albumin excretion, cardiovascular disease, and endothelial dysfunction in non-insulin-dependent diabetes mellitus. Lancet. 1992 Aug 8;340(8815):319-23.

4. Fiad TM, Freaney R, Murray B, McKenna MJ. The prevalence of microalbuminuria and associated risk factors in a population with insulin-dependent diabetes mellitus. Irish Journal of Medical Science. 1993;162 (8): 318 .

5. Gupta DK, Verma LK, Khosla PK, Dash SC. The prevalence of microalbuminuria in diabetes: a study from north India. Diabetes Res Clin Pract. 1991 May;12 (2):125-8.

6. Collins VR, Dowse GK, Finch CF, Zimmet PZ, Linnane AW. Prevalence and risk factors for micro- and macroalbuminuria in diabetic subjects and entire population of Nauru. Diabetes. 1989;38(12):1602-10.

7. Park HY, Schumock GT, Pickard AS, Akhras K. A structured review of the relationship between microalbuminuria and cardiovascular events in patients with diabetes mellitus and hypertension. Pharmacotherapy. 2003 Dec;23(12):1611-6.

8. Hillege HL, Fidler V, Diercks GF et al. Prevention of Renal and Vascular End Stage Disease (PREVEND) Study Group. Urinary albumin excretion predicts cardiovascular and noncardiovascular mortality in general population. Circulation. 2002 Oct 1;106(14): 1777-82.

9. Sarnak MJ, Levey AS, Schoolwerth AC et al. American Heart Association Councils on Kidney in Cardiovascular Disease, High Blood Pressure Research, Clinical Cardiology, and Epidemiology and Prevention. Kidney disease as a risk factor for development of 
Research Article

cardiovascular disease: a statement from the American Heart Association Councils on Kidney in Cardiovascular Disease, High Blood Pressure Research, Clinical Cardiology, and Epidemiology and Prevention. Hypertension. 2003 Nov; 42(5):1050-65.

10. Sukhija R, Aronow WS, Kakar P, Garza L, Sachdeva R, Sinha A, et al. Relation of microalbuminuria and coronary artery disease in patients with and without diabetes mellitus. The American journal of cardiology. 2006;98(3):279-81.

11. Yuyun MF, Khaw KT, Luben R, Welch A, Bingham S, Day NE, Wareham NJ; European Prospective Investigation into Cancer in Norfolk (EPICNorfolk) population study. Microalbuminuria independently predicts all-cause and cardiovascular mortality in a British population: The European Prospective Investigation into Cancer in Norfolk (EPICNorfolk) population study. Int J Epidemiol. 2004 Feb; 33 (1):189-98.
12. Mykkänen L, Zaccaro DJ, Wagenknecht LE, Robbins DC, Gabriel M, Haffner SM. Microalbuminuria is associated with insulin resistance in nondiabetic subjects: the insulin resistance atherosclerosis study. Diabetes.1998 May; 47(5): 793-800.

13. Manaviat MR, Afkhami M, Shoja MR. Retinopathy and microalbuminuria in type II diabetic patients. BMC Ophthalmol. 2004 Jul 1;4:9.

14. Skyler JS. Microvascular complications. Retinopathy and nephropathy. Endocrinol Metab Clin North Am. 2001 Dec;30(4):833-56.

15. Deckert T, Feldt-Rasmussen B, Borch-Johnsen K, Jensen T, Kofoed-Enevoldsen A. Albuminuria reflects widespread vascular damage. The Steno hypothesis. Diabetologia. 1989 Apr;32(4):219-26.

\section{How to cite this article?}

Goswani D, Jatav M, Raval A. A study of Microalbuminuria and its association with various cardiovascular risk factors and complications in patients with Type II Diabetes Mellitus.Trop J Path Micro 2017;3(2):159-167.doi: 10.17511/jopm.2017.i2.16. 